\title{
Clinical Progress Note: Decision-making for Tracheostomy Placement in Children With Neurological Impairment
}

\author{
Tressia Shaw, MD
}

Palliative Medicine, Phoenix Children's Hospital, Phoenix, Arizona; Pediatrics, University of Arizona College of Medicine, Phoenix, Arizona.

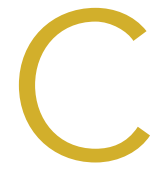

hildren with complex medical conditions are living longer, many with the help of interventions and technology, such as gastrostomy tubes, tracheostomies, ventilator support, and parenteral nutrition. Children with medical complexity and technology account for over $80 \%$ of hospital days in pediatric academic centers. ${ }^{1}$

Hospitalists need communication skills and clinical information to guide discussions with patients and families about whether to pursue these measures. Tracheostomy discussions can be particularly challenging. Over 4,000 infants and children undergo tracheostomy each year, with related hospital charges of more than $\$ 2$ billion, a 30 -day readmission rate of $24.9 \%$, and a median length of stay for pneumonia or tracheitis of 4 days. ${ }^{2}$ There is limited research on prognosis, outcomes, decision-making, and effects on quality of life, especially in the population of children who have significant neurological impairment (NI) and/or progressive or deteriorating neurological conditions. Physician biases may also influence this discussion.

This article will examine the question: How can a hospitalist guide decision-making discussions with families about tracheostomy placement for children with NI? A literature search was performed on Medline and Web of Science using the key terms tracheostomy, prognosis, neurologically impaired children, and decision-making. Articles included were relevant to the clinical question and published in the last 5 years. One article was included outside this timeframe given the scarcity of data.

\section{INDICATIONS FOR TRACHEOSTOMY}

Indications for tracheostomy include airway obstruction and the need for prolonged ventilation support. ${ }^{3}$ The number of tracheostomies placed has been increasing over the last 30 years, especially at tertiary care centers. ${ }^{3}$ Primary indications for tracheostomy include prolonged ventilation particularly in the context of underlying conditions such as congenital or acquired respiratory disease, congenital or acquired neurologic disease, cardiopulmonary disease, and primary anatomic airway obstruction. 3,4 Children who undergo tracheostomy often have multiple medical conditions that impact their overall

Corresponding Author: Tressia Shaw, MD;

Email: tshaw@phoenixchildrens.com. Telephone: 602-933-7255.

Published online first October 21, 2020.

Received: September 8, 2019; Revised: February 13, 2020;

Accepted: March 4, 2020

(c) 2020 Society of Hospital Medicine DOI 10.12788/jhm.3406 health and prognosis, with $41 \%$ having three or more complex chronic health conditions. ${ }^{5}$ This article will focus on children who have a primary indication of $\mathrm{NI}$ and in whom tracheostomy is often used as a life-prolonging measure.

\section{PROGNOSIS}

Discussions about tracheostomy should include information about risks, benefits, and prognosis. Prognosis discussions can be challenging given that many children for whom this intervention is being considered have multiple and complex medical conditions with uncertain or even known poor prognoses. Mortality rates ranging from $3 \%$ to $11 \%$ have been reported during the initial tracheostomy admission, with $\mathrm{NI}$ increasing the risk for mortality during the tracheostomy admission., 5 Children with $\mathrm{NI}$ also have higher mortality beyond the initial hospital stay, lower decannulation rates, and more frequent admissions with longer lengths of stay than do children receiving a tracheostomy for upper airway obstruction (Table 1).6,7

For most children in this population, prognosis is related more to the underlying disease process than to the risk of the surgery for tracheostomy placement itself. Discussions with families should include the anticipated prognosis of the underlying disease, as well as current available data on outcomes for children with neurological impairment who have undergone tracheostomy placement. Most patients who receive a tracheostomy are children with complex medical conditions who have an acute event that leads to airway compromise and respiratory failure underscoring the importance of advance care planning. ${ }^{5}$

\section{GOALS OF CARE DISCUSSIONS}

Clinicians face challenges when initiating advance care planning discussions, including prognostic uncertainty, the perception that families may not want to engage in these discussions, and the complexity and time these discussions can take. In one study of more than 300 chronically ill children, only $17 \%$ of parents had discussed advance directives, although $49 \%$ reported they would like to create one for their child..$^{9}$ A small study found that, although parents find these discussions difficult, they also find them important. They value a step by step approach with consideration for hope and nonmedical concerns. ${ }^{10}$ Advance care planning discussions should be viewed as a time out to clarify what the family sees as the best path forward before initiation of a tracheostomy discussion and decision.

Determining goals of care is a cornerstone of any discussion about tracheostomy placement, especially when a child has a condition that is life limiting. The decision to pursue tra- 
TABLE 1. Tracheostomy Outcomes for Patients With NI

$\begin{array}{ll}\text { Mortality } & \text { Initial in-hospital } 11.7 \% \text { vs } 3.3 \% * 6 \\ & 5 \text { year in-hospital } 8.8 \% \text { vs } 3.5 \% * 6 \\ & 14 \% \text { at } 1 \text { year and } 29 \% \text { at } 5 \text { years in moderate to severe } \mathrm{NI}^{7} \\ & 2.2-2.3 \text { higher for } 10 \text {-year study period }\end{array}$

$2.2-2.3$ higher for 10 -year study period ${ }^{* 1}$

Decannulation Rate

$4 \%$ at 1 year and $17 \%$ at 5 years in moderate to severe $\mathrm{NI}^{7}$

Length of Stay $\quad 10.3$ days vs 6.8 days $* 6$

${ }^{*}$ Comparisons are with patients who have tracheostomy for airway obstruction without NI. Abbreviation: $\mathrm{NI}$, neurological impairment.

cheostomy should involve shared decision-making. This decision-making process is the preferred communication model when multiple options could be pursued, each with its own risks and benefits. ${ }^{10}$

In this model of decision-making, the family's goals and values should be determined in the context of the medical intervention that is being pursued. Medical information such as prognosis, risk, benefits, and impact of the intervention on quality of life should all be shared with the family. Ideally, shared decision-making allows the practitioner and family to make a decision together that matches the family's goals and values with the best option available. If the family's goal is to prolong life and they feel their child has good quality of life, tracheostomy placement may be the most appropriate option. However, it is also possible that the family's goals may align more with less invasive treatment options or a transition to comfort care.

Discussions regarding goals of care can be challenging, and involving an interdisciplinary team and a Palliative Care consultant can be helpful.

\section{WHAT PROVIDERS SAY, WHAT FAMILIES NEED TO HEAR}

Research on what parents find helpful in discussions about tracheostomy is limited. One study of 56 caregivers found that parents did not feel they could make a "free choice" because the alternative to tracheostomy was death. ${ }^{11}$ In interviews with caregivers following tracheostomy, this same study found several themes in caregiver perspectives on their decision for tracheostomy (Table 2); caregivers saw a benefit to "health and well-being" from tracheostomy even though they reported feeling unprepared for the caregiving aspect at home or the potential negative side effects. Half the children in this study had a neurologic diagnosis, and only families who chose tracheostomy placement were included. To this author's knowledge, there are currently no studies that look at decisional themes, satisfaction, or outcomes for families that chose to not pursue tracheostomy.

There is limited literature about how providers discuss tracheostomy. One single-center study of practitioners found that providers focused more often on the benefits of tracheostomy rather than burdens (72\% vs $28 \%$ ). ${ }^{12}$ A common benefit theme was the provider "suggesting life with a tracheostomy might not be as difficult as families fear in that the child may have the
TABLE 2. Themes in Communication About Tracheostomy

What families say about their decision: ${ }^{11}$

What clinicians discuss with families: ${ }^{12}$

- Reported decision was based on goal to extend life and provide care at home

- Saw benefit to health after tracheostomy

- Did not feel prepared for negative effects (loss of voice, change in secretions, loss of feeding)

- Did not feel fully prepared for change in home life with tracheostomy caregiving

- Overall, reported satisfaction with their decision

- $72 \%$ Focused on benefits Faster discharge Does not have to be permanent More stable airway Allows for less sedation

- $28 \%$ Focused on burdens Requires extensive medical support May involve long-term vent dependence Presents an airway risk

ability to regain speech, engage in normal activities, and have the tracheostomy reversed once the child's health improved." However, decannulation rates and recovery trajectories for children with $\mathrm{NI}$ do not support this general expectation (Table 1). These provider communication themes may help to explain the family's perspective that they feel unprepared for the burdens of a tracheostomy or the intensity of home caregiving. Given the limited data, it is difficult to generalize. Comparing communication and decision-making themes side by side does draw attention to how providers might better communicate with families about this decision (Table 2).

The difficult aspects of caregiving deserve special attention. A study of 25 parents showed reduced parental quality of life after their child's tracheostomy placement related to overwhelming medical care, fear of death of the child requiring constant vigilance, and financial and psychological stressors. ${ }^{13}$ Most (72\%) families in this study reported decisional regret at 3 months. Resources and support for a child with this level of care varies based on the child's community. Exploration and discussion of what is available for each family, including home nursing, respite, and/or a skilled nursing facility, should be completed prior to tracheostomy placement. Honest discussions about the potential effects of this intervention on the family's life can help inform their decision.

Decision-making tools for tracheostomy could be valuable for both families and clinicians. These tools allow for a more systematic approach to the decision-making process that takes into account the multidimensional aspects of this decision. The "Child Tracheostomy Decision Guide," published by the Winnipeg Regional Health Authority, is one available tool. ${ }^{14}$ This tool guides families through the factors that may affect their decision-making and includes thoughts about goals of care, quality of life, prognosis, care at home, and other options such as comfort care. The Courageous Parents Network has also developed parent videos giving the perspective of parents who have chosen or not chosen tracheostomy. ${ }^{15}$ Currently, there are no studies that examine the usefulness of decision-making tools.

\section{GAPS IN LITERATURE}

A common theme throughout the literature is the lack of a unifying classification system for reporting outcomes data. Each study utilizes different primary indications for tracheostomy and often different definitions for NI. There is very little 
literature that focuses specifically on outcomes for children with $\mathrm{NI}$ who receive tracheostomy as a life-prolonging measure. These gaps present challenges for obtaining meaningful prognosis data to share with families. Outcomes data for children who do not receive tracheostomy is also lacking. Additional studies on how families make this decision and their decisional satisfaction could help inform the decision-making process for both parents and clinicians. Research regarding the helpfulness and outcomes with decision-making tools would be useful.

\section{CONCLUSIONS}

Although there are limited data on outcomes specific to the children with $\mathrm{NI}$ and tracheostomy, existing literature shows a higher mortality, lower decannulation rate, higher hospitalization rate, and longer length of stay than that for children who receive tracheostomy for other indications. Tracheostomy is often a life-prolonging measure for children with NI. Shared decision-making should be the preferred communication process and include defining goals of care, as well as anticipated prognosis with balanced information about risks and benefits. Further research about the decision-making process and communication would be beneficial.

Disclosure: Dr Shaw has nothing to disclose.

\section{References}

1. Children's Hospital Association. Spend for children with dominant chronic diseases - The CARE award. Historical spending: 2012-2014. https://www. childrenshospitals.org/Care/Children-With-Medical-Complexity 2018

2. Russel CJ, Mack WJ, Schrager SM, Wu S. Care variations, length of stay and readmissions in children hospitalized for bacterial tracheostomy-associated respiratory infections. Hosp Pediatr. 2017;7(1):16-23. https://doi.org/10.1542/ hpeds.2016-0104
3. McPherson ML, Shekerdemian L, Goldsworthy M, et al. A decade of pediatric tracheostomies: indications, outcomes, and long-term prognosis. Pediatr Pulmonol. 2017;52(7):946-953. https://doi.org/10.1002/ppul.23657

4. Gergin O, Adil EA, Kawai K, Watters K, Moritz E, Rahbar R. Indications of pediatric tracheostomy over the last 30 years: has anything changed? Int J Pediatr Otorhinolaryngol. 2016;87:144-147. https://doi.org/10.1016/j. ijporl.2016.06.018

5. Edwards J, Houtrow A, Lucas A, et al. Children and young adults who receive tracheostomies or were initiated on long-term ventilation in PICUs. Pediatr Crit Care Med. 2016;17(8):e324-334. https://doi.org/10.1097/ pcc.0000000000000844

6. Berry JG, Graham DA, Graham RJ, et al. Predictors of clinical outcomes and hospital resource use of children after tracheotomy. Pediatrics. 2009;124(2):563-572. https://doi.org/10.1542/peds.2008-3491

7. Tsuboi N, Ide K, Nishimura N, Nakagawa S, Morimoto N. Pediatric tracheostomy: survival and long-term outcomes. Int J Pediatr Otorhinolaryngol. 2016;89:81-85. https://doi.org/10.1016/j.ijporl.2016.07.033

8. Liberman DB, Pham PK, Nager AL. Pediatric advance directives: parents' knowledge, experience, and preferences. Pediatrics. 2014;134(2):e436-e443. https://doi.org/10.1542/peds.2013-3124

9. Lotz JD, Daxer M, Jox RJ, Borasio GD, Führer M. "Hope for the best, prepare for the worst": a qualitative interview study on parents' needs and fears in pediatric advance care planning. Palliat Med. 2017;31(8):764-771. https://doi. org/10.1177/0269216316679913

10. Nelson KE, Mahant S. Shared decision-making about assistive technology for the child with severe neurologic impairment. Pediatr Clin North Am. 2014;61(4):641-652. https://doi.org/10.1016/j.pcl.2014.04.001

11. Nageswaran S, Golden SL, Gower WA, King NMP. Caregiver perceptions about their decision to pursue tracheostomy for children with medical complexity. J Pediatr. 2018;203:354-360.e1. https://doi.org/10.1016/j. jpeds.2018.07.045

12. Hebert LM, Watson AC, Madrigal V, October TW. Discussing benefits and risks of tracheostomy: what physicians actually say. Pediatr Crit Care Med. 2017;18(12):e592-e597. https://doi.org/10.1097/PCC.0000000000001341

13. October T, Jones A, Michals H, Hebert L, Jiang J, Wang J. Parental conflict, regret, and short-term impact on quality of life in tracheostomy decision making. Pediatr Crit Care Med. 2020;21(2):136-142. https://doi.org/10.1097/ PCC.0000000000002109

14. Winnipeg Regional Health Authority. Childhood Tracheostomy Decision Guide. Accessed August 15, 2019. https://www.wrha.mb.ca/extranet/eipt/ files/EIPT-023-001.pdf

15. Courageous Parents Network. Tracheostomy Decision Making Videos. Accessed August 20, 2019. https://courageousparentsnetwork.org/video-library/decision-making/tracheostomy 